\title{
Experience with a tuberculosis antigen test in Rhodesia
}

\author{
J. B. COOKSON, J. G. CRUICKSHANK ${ }^{1}$, AND B. P. B. ELLIS
}

From the Departments of Medical Microbiology and Medicine, University of Rhodesia, Salisbury, Rhodesia

Cookson, J. B., Cruickshank, J. G., and Ellis, B. P. B. (1977). Thorax, 32, 616-618. Experience with a tuberculosis antigen test in Rhodesia. Experience with a new serological method for the diagnosis of tuberculosis is reported in a predominantly black population. We have found that in only $69 \%$ of 167 patients was there agreement between serology and the presence or absence of tuberculosis. Both false positive and false negative results were common. Of 47 healthy controls, $80 \%$ were positive. These results are less satisfactory than previous studies but differences in the reading of the results seems an unlikely explanation. Differences in the populations studied may be an important factor.

Tuberculosis is a common problem in developing countries, entering the differential diagnosis of most chest conditions. Diagnosis may be particularly difficult; Heaf testing is of limited value in populations with a high incidence of primary infection and sputum microscopy and culture requires considerable technical expertise. Thus the findings by Nicholls (Nicholls, 1975; Nicholls and Horsfield, 1976) that a simple serological method appeared to be a useful test for the disease was of great interest to us in Rhodesia. No reports have hitherto been published on the use of this test in a Black population.

In this paper we record our experience with this test on subjects in this country.

\section{Methods}

Sera were taken from 167 patients and from 41 healthy controls. Although the patients were largely those in whom tuberculosis was suspected or had been proved, some had a variety of other diseases. All those in whom tuberculosis was suspected were investigated by culture or biopsy of appropriate tissue. The diagnosis of active tuberculosis was considered proven either by positive culture or when a characteristic histological appearance, not necessarily with acid-fast bacilli, was seen in the biopsy specimen. Controls consisted of 1Present address: Public Health Laboratory, Church Lane, Heavitree,
Exeter EX2 5AD, Devon, UK
10 healthy laboratory staff members who were not investigated further and 31 food handlers at a holiday resort who underwent clinical examination and had chest radiographs performed. All controls were followed up for at least one year.

Sera were stored at $-20^{\circ} \mathrm{C}$ without preservative. Antigen was prepared by Dr. A. C. Nicholls, of the Midhurst Medical Research Institute, as previously described (Nicholls, 1975), except that Mycobacterium tuberculosis H37Rv was used $\vec{\circ}$ rather than the avirulent H37Ra strain. The agglutination tests were performed by the modified $\stackrel{x}{\circ}$ Widal technique as used by Nicholls et al. (1975). Titres were recorded as $<1 / 125,1 / 125,1 / 250$, $1 / 500$, and $1 / 1250$. A result equal to or greater than $1 / 125$ was considered positive. Seven sera, 응 of which the titres were at variance with the diagnosis, were sent to Midhurst for confirmation. Serial samples were not obtained.

The results are reported in a similar fashion to $N$ those of Nicholls and Horsfield (1976).

\section{Results}

In Table 1 subjects have been divided into five groups according to the results obtained. Group $1 \stackrel{?}{+}$ are those with both proven tuberculosis and posi- 7 tive serology; group 2 , those without proven $\stackrel{P}{\mathbb{P}}$ tuberculosis and with negative serology; group $3, \stackrel{\mathbb{Q}}{\stackrel{9}{9}}$ those with proven tuberculosis but negative $\stackrel{\mathbb{Q}}{\Omega}$ serology; and group 4, those without proven tuber- 
Table 1 Agglutination titres against M. tuberculosis antigen

\begin{tabular}{lrrllll}
\hline Titre & & Group 1 & Group 2 & Group 3 & Group 4 & Group 5 \\
\hline Negative & $<1 / 125$ & - & 14 & 29 & - & 8 \\
Positive & $1 / 125$ & 34 & - & - & 6 & 7 \\
& $1 / 250$ & 8 & - & - & 2 & 6 \\
& $1 / 500$ & 37 & - & - & 13 & 15 \\
& $1 / 1250$ & 23 & - & - & 1 & 5 \\
Total & & 102 & 14 & 29 & 22 & 41 \\
\hline
\end{tabular}

culosis but with positive serology. Group 5 are the controls.

Group 1 contains 82 patients with pulmonary tuberculosis and 20 with tuberculosis in other sites. Nine of the latter had tuberculous adenitis, five spinal tuberculosis, and two each tuberculous meningitis, pericarditis, and peritonitis. Of all 102 subjects, 91 had received antituberculosis treatment for one month or more but, of the 56 who had been treated for more than three months, 36 still had titres of $1 / 500$ or more. Thirty-eight had serial sputum cultures performed. Of 17 whose sputum had already converted to negative by the time blood for serology was taken, 13 had titres of $1 / 500$ or more. Of the 21 whose sputum converted later, 14 had similar titres.

Group 2 contains 14 patients, eight of whom had a variety of non-tuberculous diseases and six had old pulmonary tuberculosis (Table 2), in whom serology was negative.

Group 3 contains 24 patients with proven pulmonary tuberculosis, three with tuberculous adenitis, and two with miliary tuberculosis; all had negative serology. Twenty-four had received antituberculosis treatment for one month or more, 15 for more than three months. Thirteen had serial sputum cultures performed; six had converted to negative before blood for serology was taken, and seven converted later.

Table 2 Details of patients in group 2 (not proven tuberculosis and negative serology)

\begin{tabular}{|c|c|}
\hline Diagnosis & No. \\
\hline Old pulmonary tuberculosis & 6 \\
\hline Malignancy & 4 \\
\hline Squamous cell carcinoma & 1 \\
\hline Anaplastic carcinoma & 1 \\
\hline Metastatic carcinoma & 1 \\
\hline Liposarcoma & \\
\hline Congestive cardiac failure & 1 \\
\hline Bronchopneumonia & 1 \\
\hline Bronchiectasis & 1 \\
\hline Lung abscess & 1 \\
\hline Total & 14 \\
\hline
\end{tabular}

Of the 22 subjects in group 4, 10 were considered to have tuberculosis on clinical grounds and the remainder had a variety of non-tuberculous conditions. All had positive serology (Table 3). Group 5 contained 41 controls. None had developed clinical evidence of tuberculosis after a one-year follow-up period.

Identical titres were obtained in the seven paired sera which were sent to Midhurst for confirmation.

Table 3 Details of patients in group 4 (not proven tuberculosis, serology positive)

\begin{tabular}{lcccc}
\hline Diagnosis & Titres & & & \\
& $1 / 125$ & $1 / 250$ & $1 / 500$ & $1 / 1250$ \\
\hline Old pulmonary tuberculosis & 3 & - & 1 & - \\
$\begin{array}{l}\text { Pulmonary tuberculosis } \\
\text { Tuberculous meningitis }\end{array}$ & - & 2 & 3 & - \\
Pneumonia & 1 & - & 1 & - \\
Amoebic liver abscess & - & - & 2 & - \\
Nephrotic syndrome & - & - & 1 & 1 \\
Pleural effusion & 1 & - & - & - \\
Carcinoma of bronchus & 1 & - & 1 & - \\
Skin carcinoma & - & - & 1 & - \\
Osteomyelitis & - & - & 1 & - \\
Pleurisy & - & - & 1 & - \\
Total & 6 & 2 & 13 & 1 \\
\hline
\end{tabular}

\section{Discussion}

These results do not provide information as clinically useful as those of Nicholls and Horsfield (1976), who found a concordance between serology and culture results in $187(87 \%)$ subjects; only 4 $(2 \%)$ subjects had positive cultures but negative serology, and $24(11 \%)$ had negative cultures but positive serology. In only $69 \%$ of our patients was there agreement between serology and the known presence or absence of active tuberculosis even though histological evidence was accepted as proof of the diagnosis. We did not perform serial estimations and it is possible that some subjects in group 3 (proven tuberculosis but negative serology) might have developed positive titres later. Those of group 4 (no proven tuberculosis but positive serology) who had clinical evidence of tuberculosis did not have higher titres than those who did not. Some may have had tuberculosis in addition to the primary condition, but only necropsy could establish this. The finding of positive titres in $80 \%$ of apparently normal controls is further evidence of the unpredictability of the test at least in our hands.

Nicholls (personal communication) has suggested that the simple designation of a dilution of $1 / 125$ as a positive result is less valuable than a 
probability factor assigned to each dilution, this factor being the percentage of subjects with a titre of this magnitude from whom acid-alcohol-fast bacilli have been cultured. In Table 4 we have compared at each dilution the percentage of Nicholls' subjects who were culture positive with the percentage of our own subjects (excluding the controls) who were either culture or biopsy proven. It will be seen that Nicholls finds that the higher titres are the more significant and that most subjects with low titres had negative cultures. We find some evidence that high titres are more significant than low ones but also find that many subjects with low titres had tuberculosis.

Table 4 Comparison of correlations between presence or absence of tuberculosis and titre levels in Midhurst (Nicholls, personal communication) and Salisbury

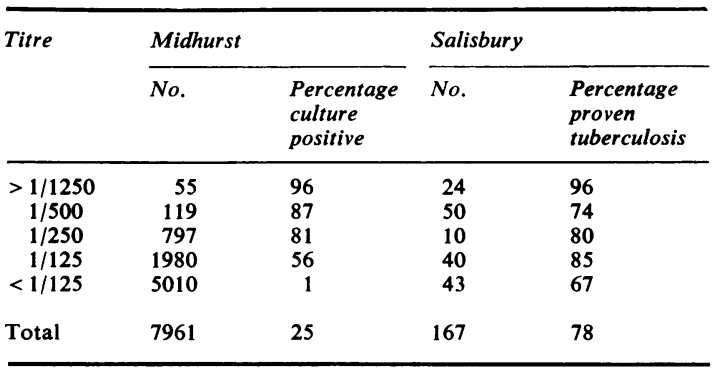

These discrepancies might be accounted for by differences in the reading of results between the two laboratories. Nevertheless there was a good correlation when paired sera were sent to Midhurst for confirmation. Over-reading might explain the results in the control group and in group 4 (positive serology but tuberculosis not proven) but not those in group 3 (negative serology but proven tuberculosis). Under-reading would give the same problem in reverse. An alternative ex-듬 planation may be the nature of the two populations. All our subjects except for the laboratory $\widehat{D}$ staff were Africans. Many, even those without proven tuberculosis, will have had a primary in-s fection. Few will have received BCG. There mayo be other unknown differences in immunological $\overrightarrow{\vec{\omega}}$ status.

Most of our patients with proven tuberculosis were receiving treatment but Nicholls and Hors- $-\frac{x}{\omega}$ field (1976) suggest that titre levels remain high, $N$ at least during the early months of treatment. In group 1 neither duration of therapy nor time of $\frac{}{\sigma}$ sputum conversion seemed to influence titre levels, $\circ$ which suggests that the negative titres in group $3_{-}^{\supset}$ cannot be explained by these factors.

We conclude that in this population the test? correlates insufficiently with the clinical state to beo useful diagnostically at the present time.

We thank Dr. N. Baker, Mrs. F. E. Brand, and Mrs. J. M. Travers-Drapes for assistance.

\section{References}

Nicholls, A. C. (1975). A serodiagnostic test for tuber

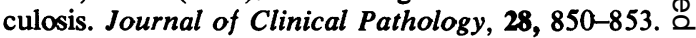

Nicholls, A. C., and Horsfield, K. (1976). Serologica 5 diagnosis of tuberculosis: a report of 12 months 3 clinical experience. Thorax, 31, 289-293.

Nicholls, A. C., Pease, P. E., and Green, I. D. (1975) Agglutinin response to bacterial infection in acute exacerbations of chronic bronchitis. Journal of Clinical Pathology, 28, 279-283.

Requests for reprints to: Dr. J. B. Cookson, Benenden Chest Hospital, Benenden, Cranbrook, Kent, UK. 